\title{
A velocity effect for representational momentum
}

\author{
JENNIFER J. FREYD \\ Cornell University, Ithaca, New York
}

and

\author{
RONALD A. FINKE \\ State University of New York at Stony Brook, Stony Brook, New York
}

\begin{abstract}
Observers were shown a rectangle at three orientations along a possible path of rotation. They were instructed to remember the third orientation in the sequence and then were presented with a rectangle at a fourth orientation that was either the same as, or slightly different from, the third orientation. Each observer tested was more likely to accept as "same" those distractors that were rotated slightly past the third orientation than those test items presented in the physically same position, and the degree of memory shift increased with increasing rate of presentation of the inducing displays.
\end{abstract}

The importance of detecting and representing movement for human perception and cognition has been noted by many researchers (e.g., Gibson, 1979; Jones, 1976). There is considerable evidence, for example, that we are more receptive to changes in the environment than to stable qualities (e.g., Johansson, 1975). Therefore, it is likely that we have acquired cognitive structures specifically developed for processing and representing dynamic information. Furthermore, it may be that those cognitive structures reflect qualities of motion normally encountered in the physical world (cf. Shepard, 1984). To the extent that this is true, we might expect to find that certain "distortions" in memory can be induced when unnatural motion is displayed. For instance, one's memory for the final position of an abruptly halted object, previously represented as moving, might be distorted so that the object is remembered as further along the path of motion than it actually was. Such a distortion would be analogous to physical momentum, or the tendency of a physically moving object to continue along its path of motion.

We previously reported (Freyd \& Finke, 1984) a finding consistent with the hypothesis that memory for the final position of an object can be distorted following observation of a sequence of displays that depict a reversible motion of a simple geometric form. Subjects were presented with three orientations of a rectangle sampled from a path of rotation about the rectangle's center, and were instructed to remember the third position. ${ }^{1}$ We then tested their memory by asking them to decide whether

The research reported here and the preparation of the manuscript were funded by a Cornell faculty research grant to the first author. We thank Michael Tarr and Ralph Hansen for laboratory assistance, Richard Darlington for advice on data analysis, and James Cutting, Douglas Elrod, Frank Keil, Carol Krumhansl, and Roger Shepard for critiques of an earlier draft of this report. Requests for reprints should be addressed to J.J. Freyd, Department of Psychology, Uris Hall, Cornell University, Ithaca, NY 14853. a fourth rectangle, presented at the same orientation as the third or rotated slightly forward or backward, was in fact at the same orientation. We found that subjects were much less likely to reject forward displacements of the rectangle than backward displacements, presumably because forward displacements were more confusable with their memory of the third position. We conjectured that memory of the third position was distorted in the direction of the implied motion. Furthermore, in a control condition, in which the order of presentation of the first two orientations of the rectangle was reversed, no asymmetry for rejection of distractor types was found, supporting the hypothesis that a consistent path of transformation must be implied in order to induce the memory distortion. However, in our initial study, we could not rule out the possibility that subjects actually remembered the true position of the third orientation but were more likely to accept forward than backward test orientations. Such a bias would be interesting, but not supportive of the notion that the actual memory for the third position is distorted in the direction of the motion.

We now report findings that more directly measure memory distortions following observation of a similar inducing display. In particular, we have found (1) evidence of a shift in the representation of the position of a form under these inducing conditions, and (2) evidence that the magnitude of that shift is a function of the implicit velocity of the inducing display. As in our earlier study (Freyd \& Finke, 1984), subjects were shown an ordered sequence of three orientations of a rectangle depicting rotation around its central axis. Again we tested their memory of the third position by having them make a same/different judgment when shown a fourth orientation of the rectangle (see Figure 1). The new procedure used a different distribution of test orientations. In the first study, 50\% of the test orientations were truly the same as the third orientation, $25 \%$ were $6^{\circ}$ forward, and $25 \%$ were $6^{\circ}$ 


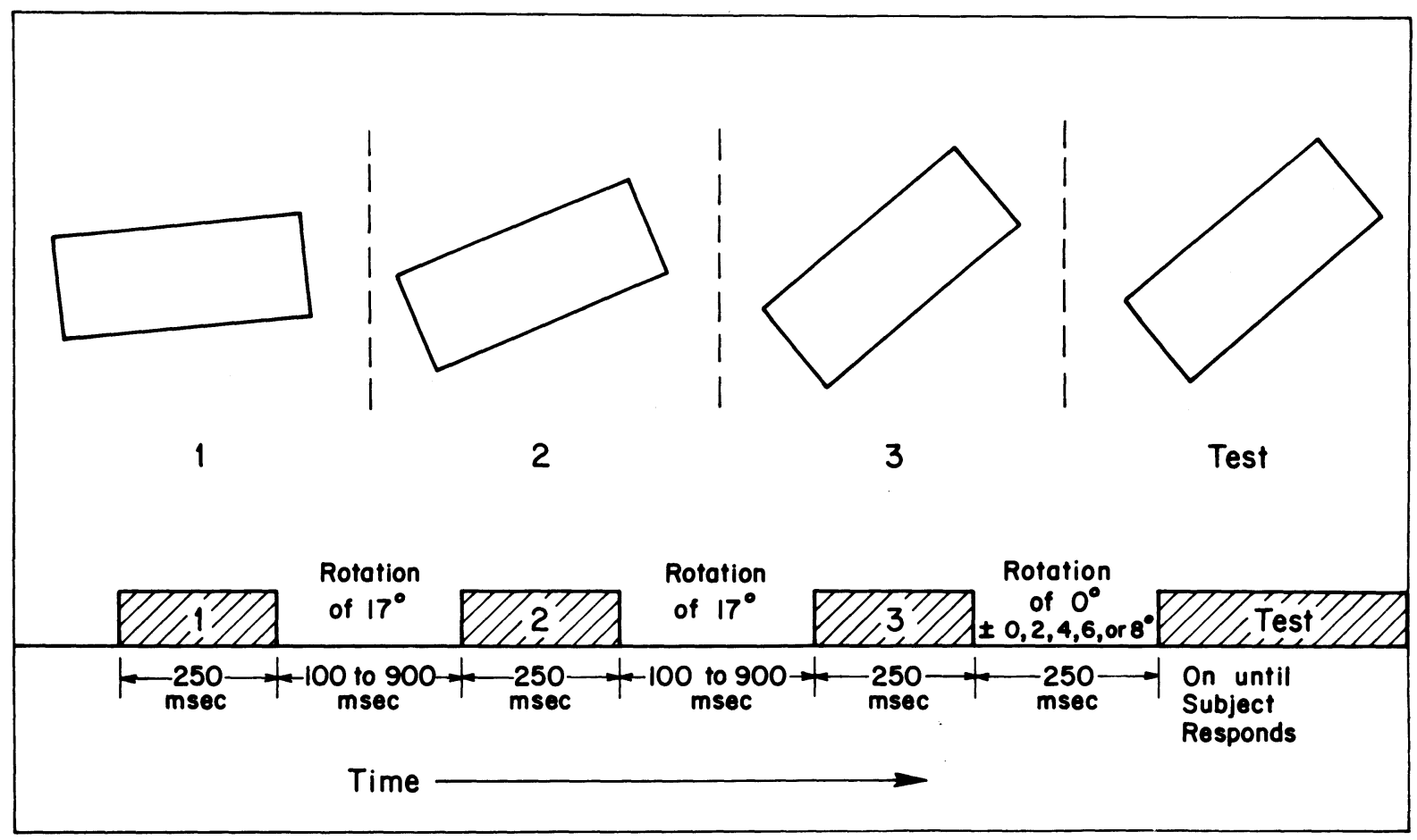

Figure 1. Example of a display sequence for an implicit counterclockwise rotation.

backward. In the new procedure, test orientations were varied parametrically: nine different test orientations were presented equally often, and only one of those orientations was truly the same.

With this new procedure, we hoped to find a shift in the representation of the third position such that some test orientations that are forward of the third position are more likely to be considered "same" than are those that are actually the same as the third orientation. Such a result would not be susceptible to the possible explanation that remains after our original study: that subjects actually remembered the true position of the third orientation but that they were more likely to accept forward than backward test orientations. If this forward-bias explanation is correct, we would expect that subjects, in our new study, would most often respond "same" for the test item that is truly the same, even if they were more likely to make errors on forward than backward trials.

In the present study we also varied the interstimulus intervals (ISIs) used in the inducing display and kept the retention interval constant. We hoped to show that the magnitude of the shift was a function of the implicit velocity of the underlying rotation, with higher velocities producing stronger effects.

\section{METHOD}

\section{Subjects}

Twenty-two Cornell undergraduates were paid for their participation in this experiment.

\section{Apparatus and Stimuli}

A computer-controlled vector-plotting HP graphics display screen was used to present stimuli. Figure 1 shows, from left to right, the three stimulus presentations for a counterclockwise rotation plus the test pattern, which were presented sequentially in the experiment. The test pattern shown on the far right is for a $0^{\circ}$ displacement, in which the testpattern orientation actually matched that of the third pattern in the sequence. Distractors consisted of identical patterns rotated $2^{\circ}, 4^{\circ}, 6^{\circ}$, or $8^{\circ}$ clockwise or counterclockwise from the third pattern. The nine different test orientations were presented equally often. The first three rectangles were always presented with a $250-\mathrm{msec}$ stimulus duration; the test rectangle remained on the screen until a response was made by the subject. There were nine conditions determining the ISIs (equally spaced from 100 to $900 \mathrm{msec}$ ) between the first and second and between the second and third orientations. ${ }^{2}$ The ISI between the third orientation and the test was always $250 \mathrm{msec}$. The angular separation between the first and second orientations and between the second and third orientations was $17^{\circ}$ for all ISI conditions and for both clockwise and counterclockwise rotations. The direction of the implied rotation was counterbalanced across subjects.

\section{Procedure}

Each subject completed two blocks of 243 trials, the first block being practice and the second providing data. The 243 trials, composed of 3 trials for each of 81 trial types (formed from 9 ISI conditions $\times$ 9 distractor positions), were randomly ordered for each subject and each block. The inducing display implied a clockwise rotation for half (11) of the subjects, and a counterclockwise rotation for the other 11 subjects. Subjects were run individually in a session that lasted about $1 \mathrm{~h}$. The subjects sat in a well-lighted laboratory room at a comfortable viewing distance from the screen, resting their dominant foot on a pedal and holding a single-button response box in each hand. They initiated each trial by pressing the foot pedal and concluded each trial by pressing one of the two response buttons (one for "same" and one for "different"). Before subjects were shown the display, they were asked to read an instruction sheet that emphasized an equal probability of seeing a "differ- 
ent" orientation that was rotated clockwise or that was rotated counterclockwise from the third position. However, they were not informed about the frequency of "same" orientations and "different" orientations. Subjects were instructed to respond "same" only when the test orientation was exactly the same as the third position.

\section{RESULTS}

Figure 2A displays the results averaged across subjects and ISI conditions for the inducing display. As predicted, subjects regarded a forward distractor position as "same" more often than they chose the truly same distractor, indicating that the subjects' memory of the third position was distorted in the direction of the implicit motion of the inducing display. This effect is extremely robust, with all 22 subjects producing a (generally symmetric) unimodal distribution of "same" responses centered on not truesame but on a forward orientation.

The location of the apex of the curve, or presumed memory shift, was estimated by first subjecting the data from all distractor positions except $-8^{\circ}$ and $-6^{\circ}$ (for which fewer than $10 \%$ of the responses were "same") to a quadratic regression. The parabolic fit $(y=71.1+$ $\left.7.17 \mathrm{x}-1.5 \mathrm{x}^{2}\right)$ accounts for $97.5 \%$ of the variance. The estimated memory shift for results averaged across ISI conditions is $2.17^{\circ}$. There were no differences between the results for the subjects who received clockwise and those who received counterclockwise inducing displays.

Curves were also fit to the data for each ISI condition through quadratic regression, and the peaks of the curves were then evaluated. The peaks were considered to be estimated memory shifts. The momentum analogy predicts that the shift should be linearly related to the implied velocity of the inducing displays. Implied velocities were calculated by dividing the distance of rotation for the inducing stimuli by the stimulus onset asynchrony (the stimulus duration plus the ISI). Figure 2B illustrates that the predicted relationship was found: $r=.90 ; p<.001$. [The correlation between shift estimates and implied velocity is actually higher $(r=.94)$ when the estimates are derived from quadratic regression using all nine distractor positions. However, the percentage of the variance accounted for by the quadratic regression is higher for each of the nine ISI conditions when data for the $-8^{\circ}$ and $-6^{\circ}$ distractor positions are dropped. Average $\mathrm{R}^{2}$ with all nine positions is $82.6 \%$; when $-8^{\circ}$ and $-6^{\circ}$ data are dropped, it is $95.6 \%$.]
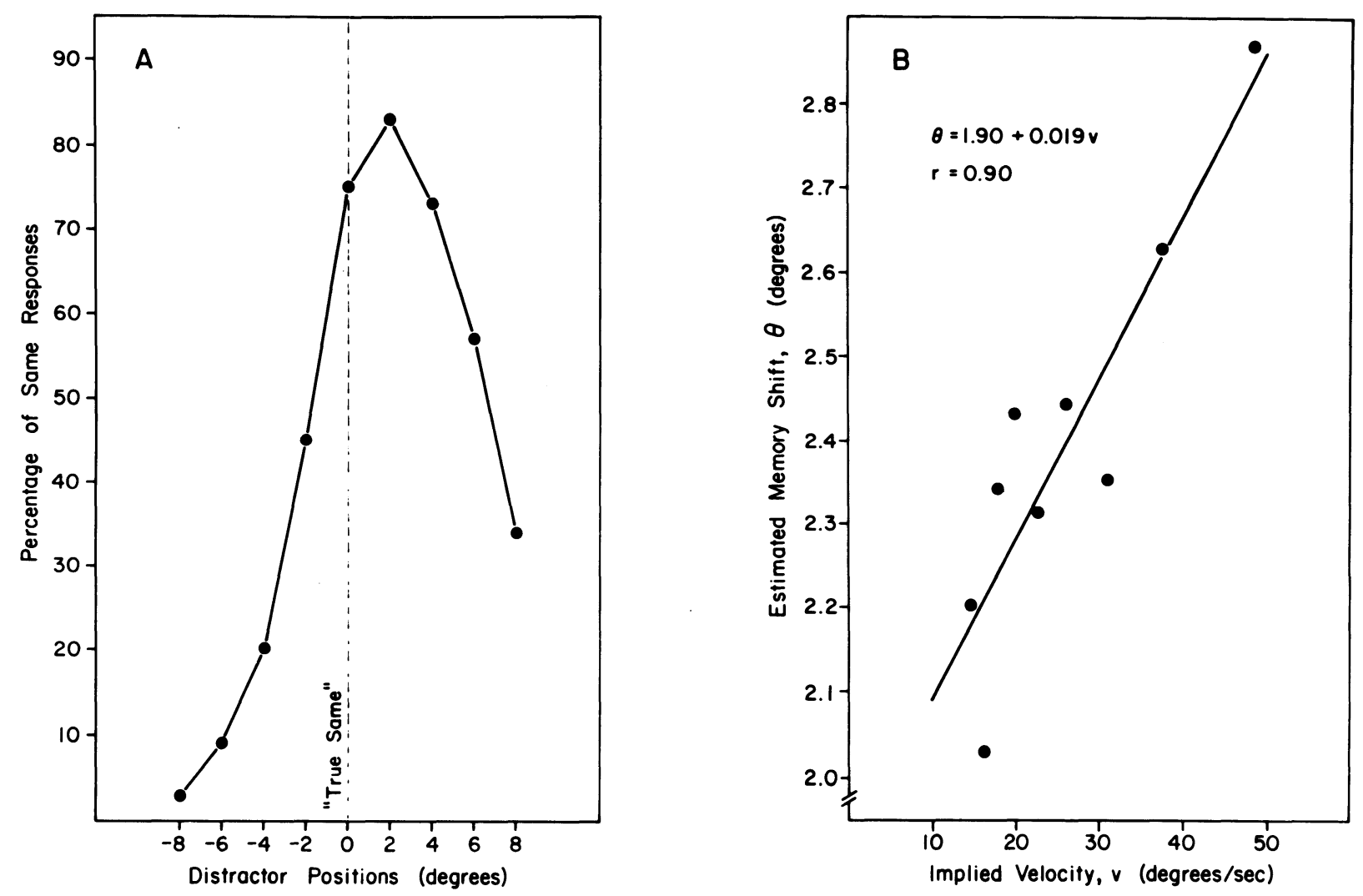

Figure 2. Results: (A) averaged across all subjects and ISI conditions. Distractor positions with positive values represent "forward" displacements of the fourth rectangle; negative values represent "backward" displacements. (B) Shift estimates derived from quadratic regressions performed on each of the ISI conditions using all distractor positions excluding $-8^{\circ}$ and $-6^{\circ}$ are plotted against implied velocity. 


\section{DISCUSSION}

The present findings, that an observer's memory for position can be distorted in the direction of implied motion and that the degree of shift is a function of the rate of that implied motion, have a number of implications. Perhaps most significantly, the present results support the general hypothesis that cognitive representations of objects and events reflect properties of the real world. In an environment in which the effects of momentum can be observed (if only before frictional forces interfere), it may be adaptive to have an "internalized" constraint on representations of moving objects that works in analogy to physical momentum. Another implication of the present findings, regarding the use of static stimuli to induce these effects, is that cognitive processes involved in detecting and representing moving objects may influence in a fundamental way the perception and memory of objects that are never actually moving (see Freyd, 1983).

The velocity effect reported here suggests numerous avenues for additional research. In one such extension, Finke, Freyd, and Shyi (1985) demonstrated a velocity effect for displays conveying translatory motion, and they discovered that observers are sensitive to the implied final velocity of the display when implied accelerating motions are used. Freyd and Johnson (1985) have begun a parametric investigation of the separate effects of implied inducing velocity and retention interval (the period of time between the third inducing stimulus and the test item); initial results suggest that the momentum effect and the velocity effect are most pronounced for fairly short retention intervals and may even change qualitatively for longer retention intervals for which long-term memory effects begin to operate. We hope that our continued investigations will uncover the nature of representational momentum and its significance for perception and memory.

\section{REFERENCES}

FINKE, R.A., \& FREYD, J. J. (1985). Transformations of visual memory induced by implied motions of pattern elements. Journal of Experimental Psychology: Learning, Memory, \& Cognition, 11, 780-794.

FINKE, R.A., FREYD, J.J., \& SHYI, G.C.-W. (1985). Implied velocity and acceleration induce transformations of visual memory. (Submitted for publication)

FREYD, J.J. (1983). The mental representation of movement when static stimuli are viewed. Perception \& Psychophysics, 33, 575-581.
FREYD, J.J., \& FINKE, R.A. (1984). Representational momentum. Journal of Experimental Psychology: Learning, Memory, \& Cognition, 10, 126-132.

Freyd, J.J., \& Johnson, J.Q. (1985). Probing the time course of representational momentum. (Submitted for publication)

GiBson, J.J. (1979). Ecological Approach to Visual Perception. Boston: Houghton Mifflin.

JoHANSSON, G. (1975). Visual motion perception. Scientific American, 232, 76-89.

JonES, M.R. (1976). Time, our lost dimension: Toward a new theory of perception, attention, and memory. Psychological Review, 83, 323-355.

Kolers, P.A. (1972). Aspects of Motion Perception. New York: Pergamon Press.

MAYHEW, J.E., \& ANSTIS, S.M. (1972). Movement aftereffects contingent on color, intensity, and pattern. Perception \& Psychophysics, 12, 77-85.

SHEPARD, R.N. (1984). Ecological constraints on internal representation: Resonant kinematics of perceiving, imagining, thinking, and dreaming. Psychological Review, 91, 417-447.

\section{NOTES}

1. We used a sequence of static displays, rather than continuously moving displays, in order to avoid residual aftereffects of motion (Mayhew \& Anstis, 1972). Such aftereffects would be in the direction opposite that of any actual motion of the inducing display; hence they might serve to suppress a "momentum" effect.

2 . Even the shortest ISIs used, and certainly the longest, are too long to induce the continuous apparent motion of a rectangle rotating. We have found that the ISIs must be reduced to less than $50 \mathrm{msec}$ to achieve a display inducing apparent motion, presumably because of the rather long (250-msec) stimulus durations of the rectangle; see Kolers (1972) on the combined effect of stimulus duration and ISI on apparent motion. In any case, Finke and Freyd (1985) have recently demonstrated that a "momentum effect" as described in Freyd and Finke (1984) can be found even with very long ISIs (on the order of $2 \mathrm{sec}$ ), suggesting that sensory mechanisms are not playing a crucial role. See Finke and Freyd (1985) for a discussion of sensory versus memory origins of the momentum effect.

(Manuscript received August 8, 1985.) 